\title{
Plasmonic Coupling Dynamics of Silver Nanoparticles in an Optical Trap
}

\author{
Marc Blattmann $^{\dagger}$ and Alexander Rohrbach ${ }^{*}{ }^{\dagger, \dagger}$ \\ ${ }^{\dagger}$ Lab for Bio- and Nano-Photonics, Department of Microsystems Engineering (IMTEK), University of Freiburg, \\ Georges-Köhler-Str.102, 79110 Freiburg, Germany \\ ${ }^{\ddagger}$ BIOSS - Centre for Biological Signalling Studies, University of Freiburg, Freiburg, Germany
}

\begin{abstract}
We investigate binding and plasmonic coupling between optically trapped $80 \mathrm{~nm}$ silver spheres using a combination of spectroscopic sensing and $3 \mathrm{D}$ interferometric laser particle tracking on a $1 \mu$ s time scale. We demonstrate that nanoparticle coupling can be either spontaneous or induced by another particle through confinement of diffusion. We reveal ultrafast entries and exits of nanoparticles inside the optical trap, fast particle rearrangements before binding, and dimer formation allowing new insights into nanoparticle self-assembly.
\end{abstract}

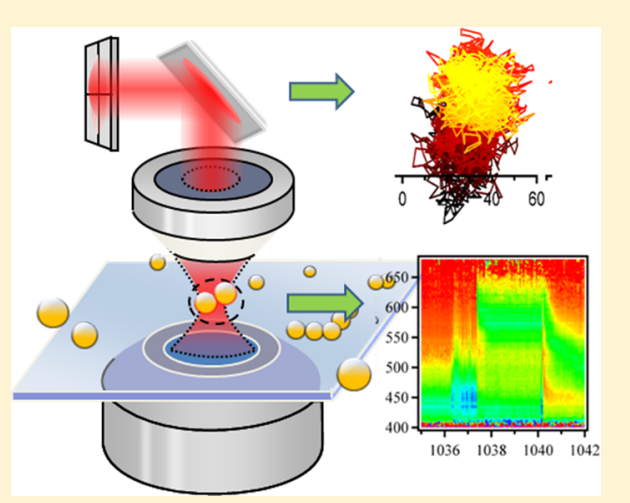

KEYWORDS: Metal nanoparticle, optical trapping, plasmonic coupling, back focal plane interferometry, reflection spectroscopy

$\mathrm{T}$ he construction of functional nanosystems by single building blocks that are assembled piecewise by a bottomup approach has long been one of the fundamental goals in nanotechnology. On this road, optical traps are considered to be a key technology to grab tiny building blocks and maneuver them precisely to the nanosystem, ${ }^{1}$ since nearly arbitrary light distributions and force fields can be generated. Even more importantly, the optical manipulation forces can be switched on and off on demand.

Among the different types of nanoscale building blocks that can be assembled, small metal spheres are of special interest, ${ }^{2-4}$ since they represent the most simple geometry, and their interaction properties with light are well understood., Spherical nanoparticles (NP) can stick together and form dimers or oligomeric structures, each revealing characteristic plasmonic resonances in their spectrum. ${ }^{7}$ For a single NP, plasmonic oscillations are most localized, and scattered light is enhanced around a single characteristic wavelengths. If NPs get into contact and form NP compounds, the resulting emission spectrum is more red-shifted, the larger the diameter of the NP compound.

Optical traps are a prime tool to investigate the plasmonic coupling of NPs, as the interaction probability is strongly increased inside the optical trap. This has been demonstrated in a variety of contexts, especially in combination with dark field microscopy. ${ }^{8-14}$ Using fast spectrometers, even the binding kinetics of two functionalized particles can be investigated in terms of their coupling times for different temperatures. ${ }^{15}$

The temperature rise of trapped NPs plays a crucial role. When the NP aggregate grows, the polarizability and absorption also increases, especially when the plasmon resonance frequency approaches the frequency of the laser light. The resulting thermophoretic forces compete with a variety of forces: optical forces, from both near-fields and farfields, ${ }^{16,17}$ temperature-dependent surface forces, ${ }^{14}$ and thermal random forces, leading all together to fluctuations in position and orientation of the NPs, which can hardly be predicted.

However, this interplay of forces, characterized by a strong stochastic component, makes the investigation of NP interaction and NP assembly difficult to observe and control.

In this paper we propose a new approach to gain further insights into the assembly and disassembly of NP inside an optical point trap. Instead of using camera based dark field microscopy, we exploit the technique of ultrafast back focal plane (BFP) interferometry, which records interferences between coherently scattered light and the incident light of the trapping laser with quadrant photo diodes (QPDs). The position-dependent intensity signals are then correlated with the spectral shifts generated by the interactions of the trapped metal NP. In this way we are able to observe fast entry and exit events of the NP, and fast changes in position and orientation resulting in different interaction paths prior to coupling.

Experimental Approach. Figure 1a displays the simplified experimental setup and sketches the measurement principle. In an inverted microscopy configuration, a $1064 \mathrm{~nm}$ continuous wave laser beam (Smart Laser Systems, Berlin) with a typical laser power of $100 \mathrm{~mW}$ is focused by an infrared water

Received: June 9, 2015

Revised: November 17, 2015

Published: November 25, 2015 


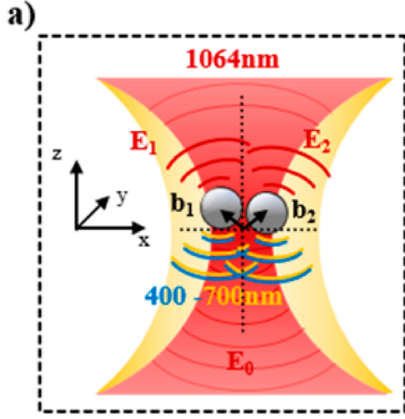

b)

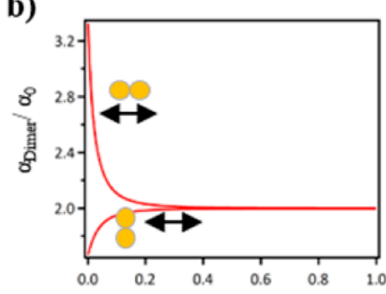

Dimer distance $[\mu \mathrm{m}]$

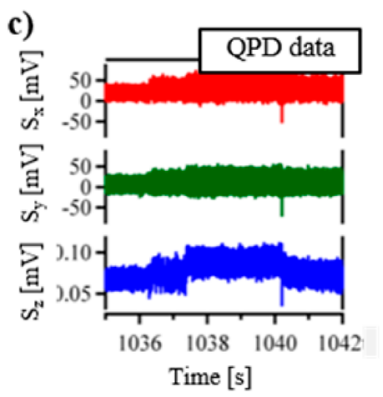

QPD

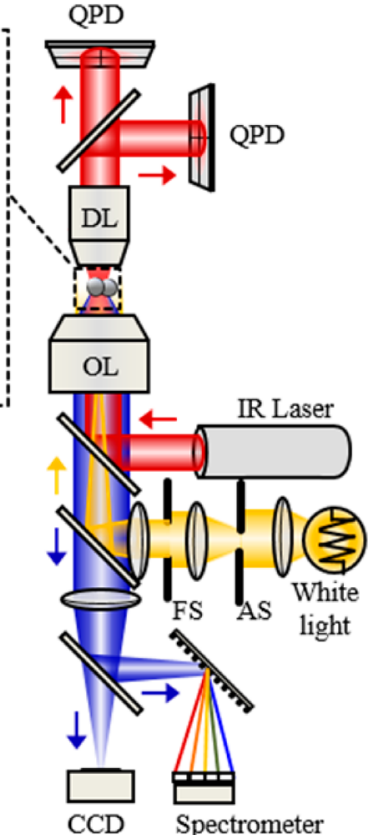

d)

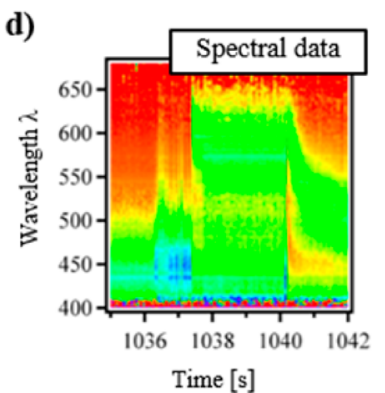

Figure 1. Optical trapping, 3D interferometric position tracking, reflection mode imaging and time- spectroscopy of metal nanospheres. (a) Sketch of the setup. Nanoparticles are trapped in water by a NIRlaser focus from a NA $=1.2$ objective lens $(\mathrm{OL})$. Interference of unscattered NIR-light and light scattered at the particles is recorded by two quadrant photo diodes (QPD) in the back-focal plane of the detection lens (DL). Collimated white light is directed through spatial selection (field stop, FS) and angular selection (aperture stop, AS) onto the trapped particles. Backscattered light is used for imaging (reflection mode) by a camera (CCD) and for time-resolved spectroscopy (SPEC). (b) Polarizability of dimers. (c) 3D tracking data from QPDs using forward scattered laser light from interacting spheres at $1 \mathrm{MHz}$. (d) Spectroscopic data from white light reflected from interacting silver NPs at $30 \mathrm{~Hz}$.

immersion objective lens (OL) with numerical aperture NA = 1.2 to form a diffraction limited focus. Silver (Ag) spheres (from BBI solution, Cardiff, UK), with a nominal diameter of $80 \mathrm{~nm}$ resulting in a resonance peak at $\lambda_{1 \mathrm{p}}=450 \mathrm{~nm}$, are optically trapped in three dimensions.

Particles are stabilized by negatively charged citrate (carboxyl) groups attached to the outer particle shell. Agglomeration is prevented by repulsive, electrostatic forces (EF), counteracting attractive van der Waals forces. EF are controlled by the ion concentration as well as the $\mathrm{pH}$-value of the embedding fluid. Using a PBS buffer as a base solution, we kept the $\mathrm{pH}$-value constant, whereas the ion concentration was adapted by diluting the buffer with ultrapure water (1:1 mixture). Nanoparticles were directly dispensed into the mixture by a volume ratio of about 1:1000.

A single Ag NP is trapped with force constants of $\kappa_{x}=4.6$ $\mathrm{pN} / \mu \mathrm{m}, \kappa_{y}=6.7 \mathrm{pN} / \mu \mathrm{m}$ and $\kappa_{z}=0.8 \mathrm{pN} / \mu \mathrm{m}$. The position of a single NP can be tracked interferometrically in $3 \mathrm{D}$ with nanometer $(\mathrm{nm})$ precision at a Megahertz rate: Unscattered trapping light and light coherently scattered in forward direction at one or several NPs is collected by a water dipping detection lens (DL, NA $=0.9)$ and projected onto one or two QPDs located in a plane conjugated to the BFP of the DL. The second QPD is overilluminated, thereby enabling improved axial interferometric tracking. ${ }^{18}$ The trapped NPs are illuminated with slightly focused, unpolarized white light from a mercury $(\mathrm{Hg})$ arc lamp $(120 \mathrm{~mW})$ to excite the particle plasmons (indicated by the yellow beam in Figure 1). Light backscattered from the particles (indicated by the blue beam) is projected by a beam splitter (20:80) onto a monochrome CCD camera and a spectrometer (Avantes AS-5216) operating at 30 $\mathrm{Hz}$.

The QPDs deliver three intensity signals, $S_{x}(t), S_{y}(t)$, and $S_{z}(t){ }^{19}$ providing orientation and position information at a sampling rate of $1 \mathrm{MHz}$ and are plotted in red green and blue in Figure 1c. The temporally changing, spectrally decomposed light emitted by the particle plasmons is normalized by the lamp spectrum and the optical system's transmission. The resulting intensity $I(\lambda, t)$ is shown in two-dimensions in pseudo colors in Figure 1d.

Important insights about the dynamics, the formation, and the disassembly of the trapped clusters can be obtained by recording both the coherently scattered laser light and the incoherently scattered white light. Whereas the laser light is decomposed in its angular spectrum by the detection lens, the light emitted by the particle plasmons is decomposed in its temporal spectrum by a spectrometer. Any rearrangement of the NP inside the laser focus results in a change in the measured intensities of the scattered light, which are shown throughout this paper. To facilitate the interpretation of the NP movements, the origin of the intensity signals is explained briefly in the following.

Theoretical Description. The particle interactions inside the optical trap are investigated by the temporal frequency spectrum of the backscattered white light, $I(\omega, t)$, and by the $2 \mathrm{D}$ spatial frequency spectrum of the forward scattered trapping light $\tilde{I}\left(k_{x}, k_{y}, t\right)$. Each intensity is the modulus square of the corresponding electric field, $\left|\mathbf{E}_{s}(\theta, \omega)\right|^{2}$, scattered at the NPs, which are assumed to be Rayleigh scatterers. The scattered electric field is characterized by its angular emission spectrum (encoded by the spatial frequency components $k_{x}$ and $k_{y}$, where $\left.k_{0} \cdot \sin \theta=\left(k_{x}^{2}+k_{y}^{2}\right)^{1 / 2}\right)$ and by the plasmonic spectrum, where $(\omega=2 \pi \mathrm{c} / \lambda)$, as well as by the perpendicular $(\perp)$ and parallel (II) field component:

$$
\left(\begin{array}{c}
E_{\| s}(\theta, \omega) \\
E_{\perp s}(\theta, \omega)
\end{array}\right)=\frac{\mathrm{e}^{-i k r}}{4 \pi r} \cdot i k^{2} \alpha(\omega)\left(\begin{array}{cc}
\cos \theta & 0 \\
0 & 1
\end{array}\right)\left(\begin{array}{c}
E_{\| \mathrm{i}} \\
E_{\perp \mathrm{i}}
\end{array}\right)
$$

This equation describes both the scattering of the broadband white light and the narrowband trapping light, defined by incident field components $E_{\| \mathrm{i}}(\theta, \omega)$ and $E_{\perp \mathrm{i}}(\theta, \omega)$ with amplitude $E_{0}$. Here, the spectral polarizability according to Clausius/Mossotti, $\alpha(\omega)=V \cdot\left(1+k_{f}\right) \cdot\left(\varepsilon_{\mathrm{s}}(\omega)-\varepsilon_{\mathrm{i}}\right) /\left(\varepsilon_{\mathrm{s}}(\omega)+\right.$ $\left.k_{\mathrm{f}} \varepsilon_{\mathrm{i}}\right)$, serves as a reasonable first approximation. $\varepsilon_{\mathrm{s}}(\omega)$ can be approximated according to Drude-Sommerfeld by $\varepsilon_{s}(\omega)=(1$ $\left.-\omega_{\mathrm{p}}^{2} / \omega^{2}\right)+i\left(\Gamma \omega_{\mathrm{p}}^{2} / \omega^{3}\right)$ using the corrected frequencies $\omega_{\mathrm{p}}=$ $0.898 \times 10^{16} \mathrm{~Hz}$ and $\Gamma=5.426 \times 10^{14} \mathrm{~Hz}$ for a $80 \mathrm{~nm} \mathrm{Ag}$ sphere. $\alpha(\omega)$ changes with the NP volume $V=4 \pi D^{3} / 6$ 


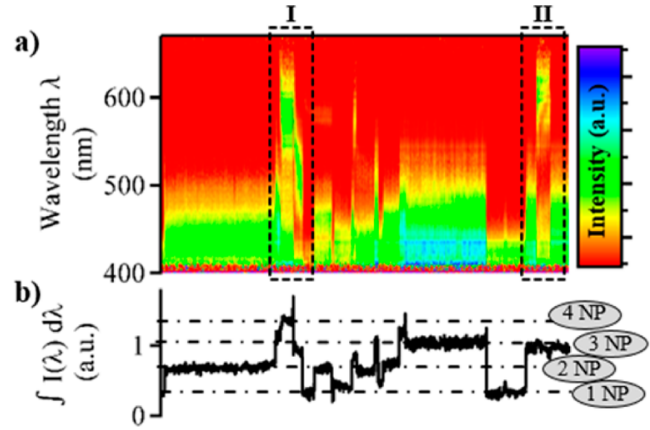

d)
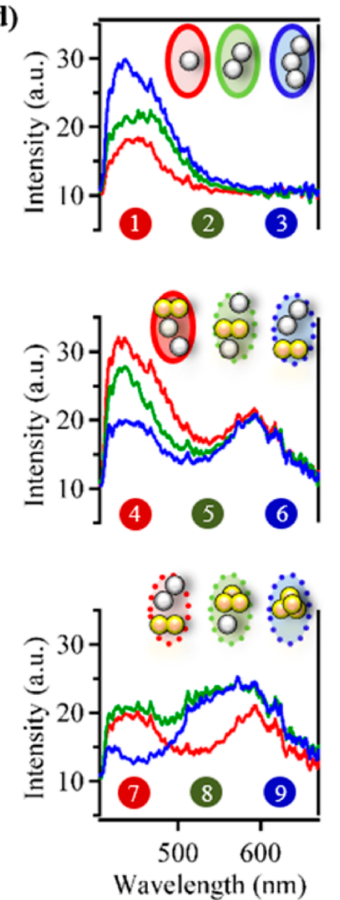

e)

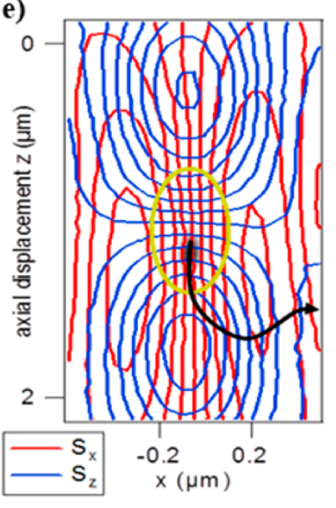

f)

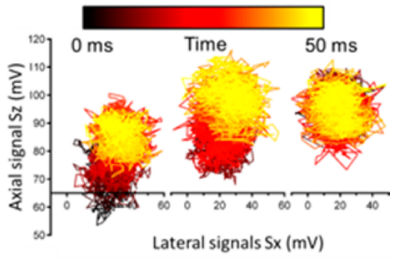

Figure 2. Combined plasmonic and interferometric sensing of particle interactions. (a) Spectral intensities $I(\lambda, t)$ vary with particle number and are due to plasmonic coupling. Region I indicates induced coupling, and region II indicates spontaneous plasmonic coupling between particles. (b) The total spectral intensity $\int I(\lambda, t) \mathrm{d} \lambda$ switches with the number of trapped particles. (c) Three interference signals $S_{x}(t), S_{y}(t)$, and $S_{z}(t)$ reveal entry and exit time points, particle displacements inside the trap, as well as particle coupling. White lines indicate averaged signals. (d) Characteristic spectra at specific time points $t_{1}-t_{9}$, for monomers $\left(t_{1}-t_{3}\right)$, for dimers $\left(t_{4}-t_{7}\right)$ and for trimers and tetramers $\left(t_{7}-t_{9}\right)$. (e) Interferometric responses $S_{x}\left(b_{x}, b_{z}\right)$, and $S_{z}\left(b_{x}, b_{z}\right)$ on particle displacements $\mathbf{b}=\left(b_{x}, 0, b_{z}\right)$ as well as the expected escape trajectory $\mathbf{b}(t)$. (f) Two-dimensional intensity trajectories for three different NP contact states in region II.

(diameter $D$ ), with the electrical permittivities of the particle and the environment, $\varepsilon_{\mathrm{s}}(\omega)$ and $\varepsilon_{\mathfrak{j}}$ and a symmetry factor $k_{\mathrm{f}}$, which is $k_{\mathrm{f}}=2$ for a sphere. Accordingly, the dipole moments $\mathbf{p}$ $=\alpha \epsilon_{0} \epsilon_{i} \mathbf{E}_{i}$ and the extinction cross-section for Rayleigh scatterers $C_{\text {ext }}(\omega)=C_{\text {sca }}(\omega)+C_{\text {abs }}(\omega)=\left(k^{4} / 6 \pi\right)|\alpha(\omega)|^{2}+k$. $\operatorname{Im}\{\alpha(\omega)\}$ are defined. $C_{\text {sca }}(\omega)$ is proportional to the intensities displayed in Figure $1 \mathrm{~d}$ and the following figures.

Plasmonic Coupling. If two particles are close to each other, the dipole moment of one particle is altered by the electric near field $\mathbf{E}_{\mathrm{nf}}$ of the other particle such that the dipole moment of particle 1 or 2 is $\mathbf{p}_{1 / 2}=\alpha \epsilon_{0} \epsilon_{i}\left(\mathbf{E}_{\mathrm{i} 1 / 2}+\mathbf{E}_{\mathrm{nf}, 2 / 1}\right)$. The combined dipole moment $\mathbf{p}_{1}+\mathbf{p}_{2}=\alpha_{D} \epsilon_{0} \epsilon_{\mathrm{i}} \mathbf{E}_{\mathrm{i}}$ results in an effective polarizability $\alpha_{\mathrm{D}}$ of a dimer, which is approximated by two particles in a center distance $d$ :

$$
\alpha_{\mathrm{D}}(\omega, d)=\frac{2 \alpha(\omega)}{1-\alpha(\omega) \cdot s / 4 \pi d^{3}}
$$

One finds $s=2$ for the dimer's longitudinal plasmon mode and $s=-1$ for its transversal mode. The polarizability of both modes are plotted in Figure $1 \mathrm{~b}$ as a function of the particle distance $d$. The corresponding spectral shift of the resonance peak also depends on $d$ and can be approximated by $\varepsilon_{\text {res }}(d)=$ $\varepsilon_{\mathrm{i}}\left(8 d^{3}+D^{3}\right) /\left(4 d^{3}-D^{3}\right)$. If more particles come into contact, the dipole moment of each particle is affected by the near fields of the other particles, and corresponding expressions for the polarizability can be derived.

Coherent Tracking Signals. As illustrated in the inset of Figure 1a, several particles with $3 \mathrm{D}$ displacements $\mathbf{b}_{1}, \mathbf{b}_{2}, \ldots$ from the center of the focus, generate the scattered electric fields $\mathbf{E}_{1}, \mathbf{E}_{2}, \ldots$ which all depend on the particle center positions $\mathbf{b}_{1}, \mathbf{b}_{2}, \ldots$. The resulting interference intensity in the BFP of the detection lens (DL) is recorded by the QPD. ${ }^{19}$ The resulting position signals in direction $j=x, y, z$ can be expressed as

$$
\begin{aligned}
S_{j}\left(\mathbf{b}_{1}, \mathbf{b}_{2}, \ldots\right) & =\iint_{\mathrm{QPD}}\left|\tilde{E}_{0}+\tilde{E}_{1}+\tilde{E}_{2}+\ldots\right|^{2} \cdot H_{j} \mathrm{~d} k_{x} \mathrm{~d} k_{y} \\
& =\iint_{\mathrm{QPD}} \sum_{n \neq m}\left(\tilde{I}_{n n}+\tilde{I}_{n m} \cos \left(\Delta \varphi_{n m}\right)\right) \cdot H_{j} \mathrm{~d} k_{x} \mathrm{~d} k_{y}
\end{aligned}
$$

where the angular spectrum of the field $\tilde{\mathbf{E}}\left(k_{x}, k_{y}, \mathbf{b}\right)=$ $\operatorname{FT}[(\mathbf{E}(x, y, \mathbf{b}))]$ is obtained by a two-dimensional Fourier transform, such that $\tilde{\mathbf{E}}_{\mathbf{0}}$ is the incident field spectrum. The functions $H_{x}$ and $H_{y}$ reflect the signal differences between each two adjacent quadrants of the QPD, and $H_{z}=1$ in the axial direction. For NP displacements smaller than half the radius of the laser focus, eq 3 provides the unique BFP interferometric position signal for a single particle $(n=1)$, i.e., $\left|\tilde{\mathbf{E}}_{\mathbf{0}}+\tilde{\mathbf{E}}_{\mathbf{1}}(\mathbf{b})\right|^{2}$. For $n>1$ particles, only position changes can be detected, without knowing the exact particle positions.

Relaxation of Signal Correlations. The cross-correlation function $\mathrm{CCF}_{j k}(\tau)$ between the QPD signals $S_{j}(t)$ and $S_{k}(t)$ in direction $j, k=x, y, z$ can be approximated by an exponential with a relaxation time $\tau_{j k}$, when the NP assembly diffuses within a minimum of a multiwell potential landscape. We approximate the restoring forces that drive the system to a stable position and orientation ${ }^{20}$ to be linear:

$$
\mathrm{CCF}_{j k}(\tau)=\left\langle S_{j}(t) \cdot S_{k}(t+\tau)\right\rangle \approx\left\langle S_{j} \cdot S_{k}\right\rangle \cdot \exp \left(-\tau / \tau_{j k}\right)
$$


The change in strength of the linear restoring forces can be estimated by the fluctuation width for each degree of freedom (DOF)

$$
\sigma_{j}=\sqrt{\left\langle S_{j}^{2}\right\rangle} \approx \sqrt{k T / \kappa_{j}}
$$

Here we assume thermal equilibrium for periods $10 \times$ longer than the relaxation time. $k T \approx 4 \mathrm{pN} \cdot \mu \mathrm{m}$ is the thermal energy at $T=300 \mathrm{~K}$.

Measurement Principles. A typical experimental situation is illustrated by Figure 2, where up to four $80 \mathrm{~nm} \mathrm{Ag} \mathrm{NPs}$ diffuse inside an optical point trap. Within a time window of 90s, NPs enter, come into contact, and exit the trap several times. The changes in relative NP distances become apparent in Figure $2 \mathrm{a}$, where the spectral change of the backscattered intensity $I(\lambda, t) \sim C_{\mathrm{sca}}(\lambda, t)=\left(k^{4} / 6 \pi\right)\left|\alpha_{\mathrm{D}}(2 \pi c / \lambda, t)\right|^{2}$ is displayed over time. The spectrum ranges from $\lambda=400 \mathrm{~nm}$ to $\lambda=650$ $\mathrm{nm}$, which is enough to identify the relevant resonance peaks of the plasmons. Figure $2 \mathrm{~b}$ shows the integrated scattered intensity $I_{\text {sum }}(t)=\int I(\lambda, t) \mathrm{d} \lambda$, which is approximately proportional to the number of NPs in the Rayleigh scatterer regime. The minimum of $I_{\text {sum }}$ can be identified by the switch between $I_{\text {sum }}=0$ and $I_{\text {sum }}>0$, when isolated particles enter or exit the trap. The three interferometric signals $S_{j}(t)(j=x, y, z$; see eq 3$)$ shown in Figure $2 c$ are synchronized in time with $I(\lambda, t)$. The changes in the fluctuation widths (standard deviations) $\sigma_{j}(t) \approx \sqrt{k T / \kappa_{j}}(t)$ are clearly visible, as well as the mean values $\left\langle S_{j}\right\rangle(t)$, which are obtained by a running average over $1 \mathrm{~ms}$ and which are displayed by the white central line.

In the exemplary experiment summarized in Figure $2 a-c$, two particles diffuse inside the optical trap without coupling, which is revealed by the intensity maximum at $\lambda_{01}=450 \mathrm{~nm}$, which is the resonance frequency of a single $80 \mathrm{~nm} \mathrm{Ag} \mathrm{NP}$. Figure $2 \mathrm{~d}$, taken from a different experiment, reveals that the shape of the spectrum hardly changes for $N=1,2$, or $3 \mathrm{NPs}$ inside the trap, while the total intensity $\int I(\lambda, t) \mathrm{d} \lambda$ increases. The insets depict uncoupled particles in white shading, whereas particles in close contact are shaded in gold, as displayed by the spectra numbered with (4)-(9). The individual spectra in Figure $2 \mathrm{~d}$ reveal a second resonance at $\lambda_{02}=600 \mathrm{~nm}$, when two particles are coupled, and, an enhanced emission around $\lambda_{03}=$ $525 \mathrm{~nm} \approx 1 / 2\left(1^{2}+2^{2}\right)^{1 / 2} \cdot \lambda_{02}=1 / 2 \sqrt{3} \cdot \lambda_{02}$, when three (or four) particles couple. The disappearance of the resonance at $\lambda_{01}$ from spectrum (8) to (9) indicates that no individual NP is present and trimers or tetramers have formed.

Events of NP entries and exits can be identified by the peaks in the position signals $S_{j}(t)$, which are visible in either one, two, or all three time traces. These peaks coincide with a change in the total intensity $I_{\text {sum }}$ and in the spectrum and can be further analyzed by the signal response patterns as shown in Figure 2e. Here, contour lines of equal $z$-signals (in blue) are overlaid with lines of equal $x$-signals, $\mathrm{S}_{x}\left(\mathrm{~b}_{x}, \mathrm{~b}_{z}\right)$ and $\mathrm{S}_{z}\left(\mathrm{~b}_{x}, \mathrm{~b}_{z}\right)$. From these lookup tables, $3 \mathrm{D}$ entry and exit paths of the NPs can be recovered. Figure $2 \mathrm{f}$ displays three $2 \mathrm{D}$ signal traces of three different NP contact states within a time window of $50 \mathrm{~ms}$ at time $1091.5 \mathrm{~s}, 1093.1 \mathrm{~s}$, and $1093.58 \mathrm{~s}$.

Two characteristic regions, marked by I and II, reveal a strong spectral red shift during NP coupling and a distinct increase in the axial position signal $S_{z}(t)$ (see arrows). The signal step results from an increased absorption and radiation pressure of the dimer, thereby pushing the dimer in the axial direction.
Spontaneous and Induced Plasmonic Coupling. The regions I and II marked in Figure 2a, are further analyzed in Figure 3. Here, two different situations are described prior to

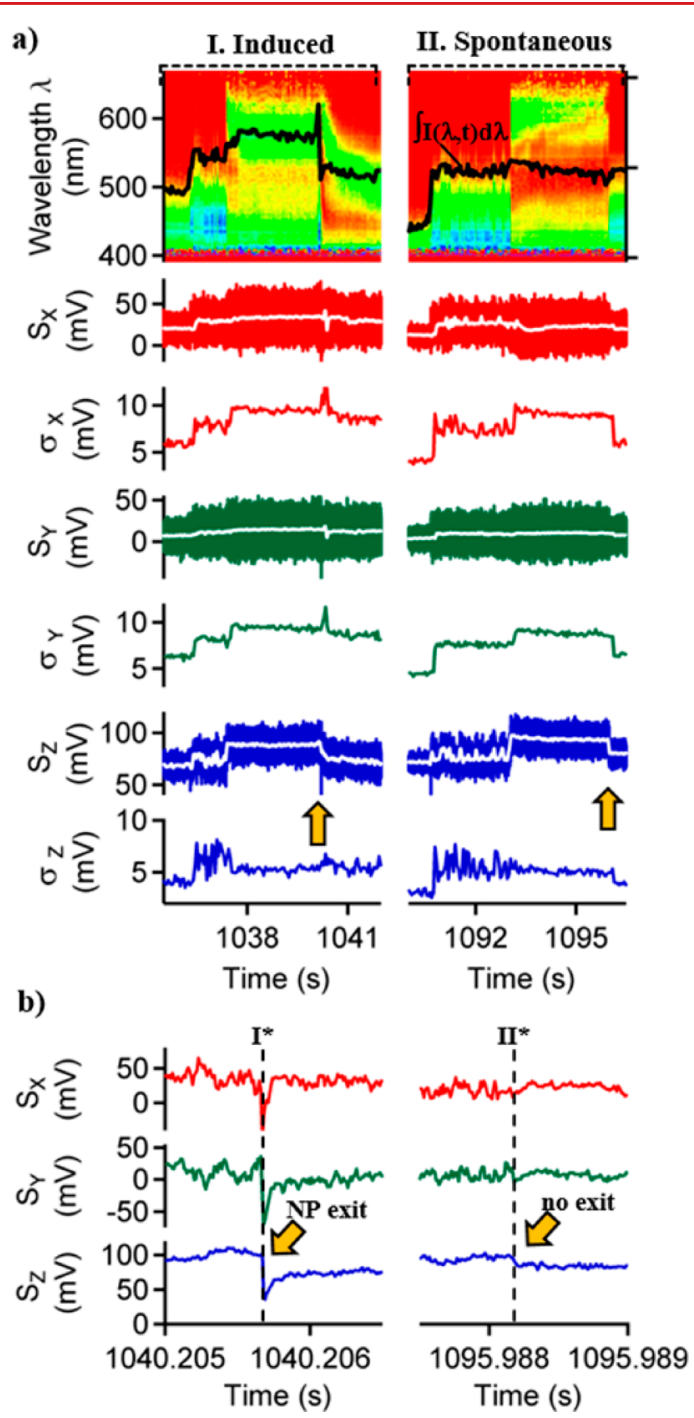

Figure 3. Nanoparticle entry and escape from the optical trap. (a) Induced and spontaneous plasmonic coupling of NP can be distinguished by spectral changes $I(\lambda, t)$, by changes in interference signals $S_{x}(t), S_{y}(t)$, and $S_{z}(t)$ and by the fluctuation widths $\sigma_{\mathrm{sx}}(t), \sigma_{\mathrm{sx}}(t)$ and $\sigma_{\mathrm{sx}}(t)$. (b) Interference position signals on a microseconds timescale during induced (I) and spontaneous (II) stop of plasmonic coupling.

coupling, which have been observed in several experiments. In one case, plasmonic coupling can be induced by the entry of further particles, which is visible in the top graph of Figure $3 a$, where the spectrum $I(\lambda, t)$ and the total intensity $I_{\text {sum }}=\int I(\lambda, t)$ $\mathrm{d} \lambda$ (black line) are plotted in one graph. Here, a third and fourth particle enter the trap (indicated by white arrows), thereby reduce the diffusion volume of all NPs and increase the contact probability of the particles. In the example, the coupled state remains stable for about $3 \mathrm{~s}$, before a fifth particle enters the trap and makes the complex unstable, leading to a fast exit of 1 or 2 particles. In the other case (right column), the coupling between two particles happens spontaneously, since no further particles enter the trap to reduce the diffusion volume. The total intensity $I_{\text {sum }}$ remains constant, no 
interferometric entry or exit signals are visible, but the spectrum shows a pronounced red shift.

Our NIR-trapping wavelength is far from the NP resonance and thereby results in significantly less heating than in a $532 \mathrm{~nm}$ optical trap, although two photon absorption processes cannot be excluded. A local temperature increase of $>100 \mathrm{~K}$ can occur without bubble forming. ${ }^{21}$ In the presence of an insulating vapor layer, however, much higher temperatures of the NPs are possible, which leads to thermal expansion of the NPs. This can lead to explosion-like transport processes of the NPs or fractions thereof. ${ }^{22}$ It is possible that such forces drive the fast escape, which is analyzed in more detail by traces with $1 \mu \mathrm{s}$ time resolution as shown in Figure $3 \mathrm{~b}$. Assuming a particle escape distance of half the focus diameter $(\Delta r \approx 0.4 \mu \mathrm{m})$ and measuring the exit time of $1 \mu \mathrm{s}$ or shorter, we estimate a very high mean exit velocity of at least $0.4 \mathrm{~m} / \mathrm{s}$. This corresponds to a mean friction force of $F_{\gamma}>0.5 \mathrm{nN}$ and a minimal mean energy accumulated inside the trap of $W_{\gamma}=F_{\gamma} \cdot \Delta r>0.2 \times 10^{-15} \mathrm{Nm}$, which is $0.5 \times 10^{5} k_{\mathrm{B}} T$ at $T=300 \mathrm{~K}$. However, further research is required to understand the local energy accumulation and the NP exits in more detail. ${ }^{22}$

In the case of spontaneous NP coupling, NP decoupling was also spontaneous, resulting in an instantaneous spectral blueshift, while the number of NPs remained constant, $\int I(\lambda, t) \mathrm{d} \lambda=$ const. In the case of induced plasmonic NP coupling, the continuous spectral blue shifting was observed in only some experiments. This blue shift might be a consequence of a slow unbinding of the remaining (three) particles or of the local temperature increase leading to a change in electric permittivities. However, such effects need to be investigated in the future as well.

By observing the three interference signals $S_{j}(t)$ (Figure $3 \mathrm{~b}$ ) and the corresponding fluctuation widths $\sigma_{j}(t)$ (see eq 5), it can be seen that all $\sigma_{j}(t)$ increase with the number of NP inside the trap. Comparing the mean signals $\left\langle S_{x}\right\rangle(t)$ and $\left\langle S_{y}\right\rangle(t)$, one finds that the temporal changes are stronger in the $x$-direction, which is the polarization direction of the trapping light. In this direction, the laser focus is slightly broader and thereby facilitates the formation and orientation of dimers along $x .^{23}$ The most pronounced signal changes are in the axial $z$ direction, where the optical trap is weakest. Again, the strong increase in $S_{z}(t)$ results from the fact that the radiation pressure

$$
F_{\text {scat }}(d) \sim\left(\frac{k_{n}^{4}}{6 \pi}\left|\alpha_{\mathrm{D}}(d)\right|^{2}+k_{n} \operatorname{Im}\left\{\alpha_{\mathrm{D}}(d)\right\}\right)
$$

for a dimer is stronger than that of two monomers, since $\alpha_{\mathrm{D}}(d$ $=D)>2 \cdot \alpha_{\mathrm{D}}(d \rightarrow \infty)$ (see Figure 1 and eq 2 ). Remarkably, the interference signals change strongly prior to coupling, which means that the NPs rearrange on a short time scale, to find the best interaction state. This can be seen best by the changes of $\sigma_{z}(t)$. Apparently, NP contacts are hindered by ongoing changes of optical, thermophoretic and surface forces.

Figure 4 shows a further analysis of the NP dynamics in terms of their cross-correlation functions CCF (eq 4). Here, four distinct arrangements of NPs are selected from the $90 \mathrm{~s}$ time window as indicated by the dashed lines numbered by $(1)-(4)$ in Figure $4 \mathrm{a}$. For a single NP at time $t_{1}$, all three $\mathrm{CCF}_{j k}(\tau)$ are relatively flat in amplitude, proving that movements in one direction hardly affect the interferometric position signals in the other direction (Figure $4 b$ ). This is not the case, when two or more particles move inside the trap and position signals couple, which can be seen by a stronger a)

b)

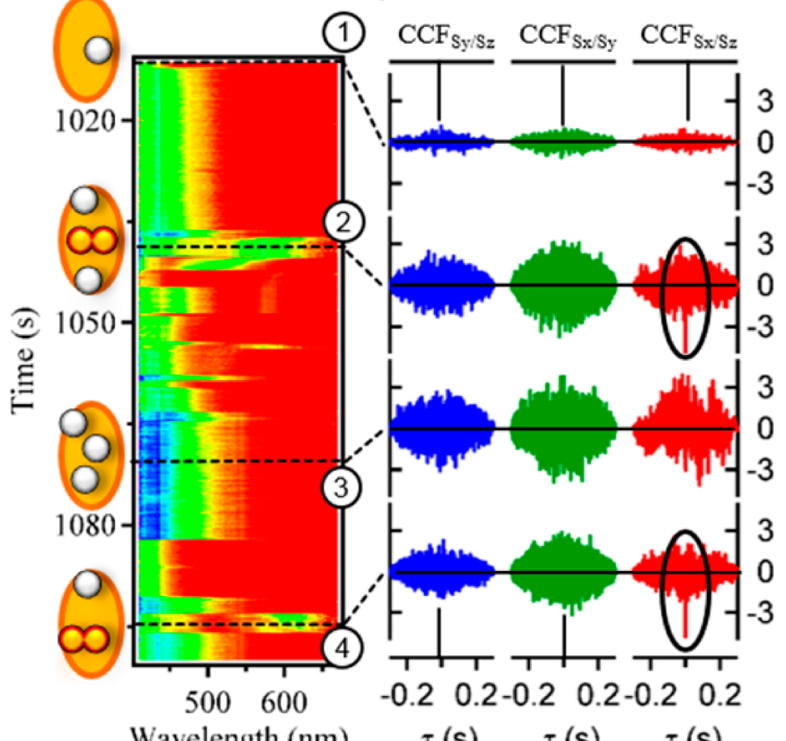

c)
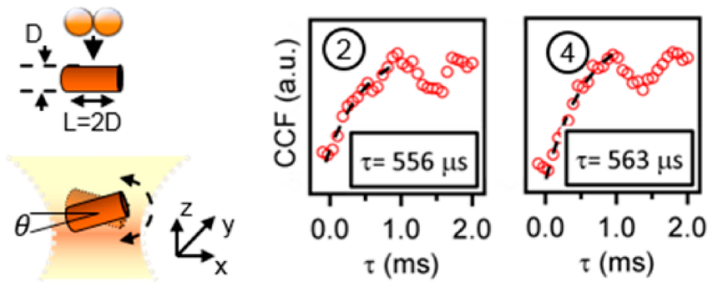

Figure 4. Formation and thermal motion of dimers. (a) The number of the trapped particles is sketched on the left for four distinct time points and scales with the total spectral intensity $\int I(\lambda, t) \mathrm{d} \lambda$. (b) The three cross-correlation functions $\mathrm{CCF}_{j k}(\tau)$ of the QPD-signals are small in amplitude for a single particle and larger for three and four particles. When particles form dimers (time points (2) and (4)), pronounced peaks in the $\operatorname{CCF}(\tau)$ in the $x z$-plane occur. (c) Measured relaxation times of about $0.5 \mathrm{~ms}$ from rotational motion of the rod-like dimer indicates its stable horizontal orientation due to the optical forces increased by plasmonic coupling.

amplitude in all three $\mathrm{CCF}_{j k}(\tau)$. However, at time points $t_{2}$ and $t_{4}$, when NPs form a dimer, the $\mathrm{CCF}_{x z}(\tau)$ (shown in red) reveal a significant negative correlation for movements in the $x z$ plane. Figure $4 \mathrm{c}$ reveals decays of $\mathrm{CCF}_{x z}(\tau)$ with a relaxation time of $\tau_{x z} \approx 0.5 \mathrm{~ms}$, which corresponds to a rotational motion relaxation of the dimer back to the horizontal orientation, indicated by the increased $S_{z}$ signal (Figure 2c) due to enhanced radiation pressure. The torque $M=\kappa_{\theta} \cdot \theta$ restoring the dimer can be estimated by the measured relaxation time $\tau_{x z}=$ $\gamma_{\theta} / \kappa_{\theta}$, with rotational viscous $\operatorname{drag} \gamma_{\theta}=\left(\pi \eta L^{3}\right) /(3 \ln (L / D)-$ 0.2 ) according to eq 4 . For a dimer length of $L=2 \mathrm{D} \approx 160 \mathrm{~nm}$, and $\eta$ being the viscosity of water at about $310 \mathrm{~K}$, this results in a rotational stiffness $\kappa_{\theta}=14 \mathrm{~nm} \cdot \mathrm{pN} / \mathrm{rad}$ and a small torque of $M \approx 5 k_{\mathrm{B}} T$ for a $90^{\circ}$ rotation. It is also possible that the two particles in contact slide against each other, if the surface friction forces are smaller than the hydrodynamic friction forces.

In conclusion, we have presented a new measurement method that allows one to observe the dynamics of interacting metal particles in a $3 \mathrm{D}$ optical trap by combining two timeresolved spectral detection systems. We used a commercial grating spectrometer operating at $30 \mathrm{~Hz}$ to measure time frequency shifts due to plasmonic coupling of $80 \mathrm{~nm}$ silver NP, 
which was synchronized with the coherent scattering signals used in back focal plane (BFP) interferometry, where spatial frequency changes are recorded by $2 \mathrm{QPDs}$ at a $1 \mathrm{MHz}$ rate. This combined detection scheme facilitates the identification of highly dynamic NP interactions, including the entry and exit of $\mathrm{NP}$, the formation of aggregates, and the orientation of dimers inside the optical trap. We could distinguish between a spontaneous and an induced formation of dimers even in the presence of other NPs. Plasmonic coupling is induced by the entry of further NPs, which reduce the diffusion volume of the trapped NPs and thereby enhance the contact probability. Fluctuation analysis through time varying fluctuation widths and signal correlations reveal a fast rearrangement of particles prior to coupling, giving insights to the changing optical, thermophoretic, and surface forces. For example, we could estimate quantities such as an ultrafast NP exit speed of $0.4 \mathrm{~m} / \mathrm{s}$, an exit force of $>0.5 \mathrm{nN}$, exit energies in the order of $10^{4} \mathrm{kT}$, or a dimer rotational stiffness of $14 \mathrm{~nm} \cdot \mathrm{pN} / \mathrm{rad}$.

By a further extension of our technique presented here, it should be possible to observe the motion of individual NPs and their aggregation in $3 \mathrm{D}$ with high precision and on very short time scales, which will help to construct functional metal nanosystems in a bottom-up approach.

\section{AUTHOR INFORMATION}

\section{Corresponding Author}

*E-mail: rohrbach@imtek.de.

Notes

The authors declare no competing financial interest.

\section{ACKNOWLEDGMENTS}

We thank Peter Piechula, Dr. Maria Dienerowitz, and Dr. Peter Reece for helpful comments on the manuscript.

\section{REFERENCES}

(1) Maragò, O. M.; Jones, P. H.; Gucciardi, P. G.; Volpe, G.; Ferrari, A. C. Nat. Nanotechnol. 2013, 8, 807-19.

(2) Svoboda, K.; Block, S. M. Opt. Lett. 1994, 19, 930-932.

(3) Hansen, P. M.; Bhatia, V. K.; Harrit, N.; Oddershede, L. Nano Lett. 2005, 5, 1937-1942.

(4) Bosanac, L.; Aabo, T.; Bendix, P. M.; Oddershede, L. B. Nano Lett. 2008, 8, 1486-91.

(5) Saija, R.; Denti, P.; Borghese, F.; Maragò, O. M.; Iatì, M. A. Opt. Express 2009, 17, 10231-10241.

(6) Tanabe, I.; Tatsuma, T. Nano Lett. 2012, 12, 5418-5421.

(7) Barrow, S. J.; Wei, X.; Baldauf, J. S.; Funston, A. M.; Mulvaney, P. Nat. Commun. 2012, 3, 1275.

(8) Prikulis, J.; Svedberg, F.; Käll, M.; Enger, J.; Ramser, K.; Goksör, M.; Hanstorp, D. Nano Lett. 2004, 4, 115-118.

(9) Ohlinger, A.; Nedev, S.; Lutich, A. A.; Feldmann, J. Nano Lett. 2011, 11, 1770-1774.

(10) Pearce, K.; Wang, F.; Reece, P. J. Opt. Express 2011, 19, 25559.

(11) Ni, X.; Emani, N. K.; Kildishev, A. V.; Boltasseva, A.; Shalaev, V. M. Science 2012, 335, 427-427.

(12) Dienerowitz, M.; Gibson, G.; Dienerowitz, F.; Padgett, M. J. Opt. 2012, 14, 045003.

(13) Ling, L.; Huang, L.; Fu, J. X.; Guo, H. L.; Li, J. F.; Ou-Yang, H. D.; Li, Z. Y. Opt. Express 2013, 21, 6618-6624.

(14) Tong, L. M.; Miljkovic, V. D.; Johansson, P.; Kall, M. Nano Lett. 2011, 11, 4505-4508.

(15) Osinkina, L.; Carretero-Palacios, S.; Stehr, J.; Lutich, A. A.; Jäckel, F.; Feldmann, J. Nano Lett. 2013, 13, 3140-3144.

(16) Demergis, V.; Florin, E.-L. Nano Lett. 2012, 12, 5756-5760.

(17) Yan, Z. J.; Shah, R. A.; Chado, G.; Gray, S. K.; Pelton, M.; Scherer, N. F. ACS Nano 2013, 7, 1790-1802.
(18) Friedrich, L.; Rohrbach, A. Opt. Lett. 2010, 35, 1920-1922.

(19) Grießhammer, M.; Rohrbach, A. Opt. Express 2014, 22, 61146132.

(20) Tränkle, B.; Speidel, M.; Rohrbach, A. Phys. Rev. E 2012, 86, 021401-1-021401-5.

(21) Bendix, P. M.; Reihani, S. N. S.; Oddershede, L. B. ACS Nano 2010, 4, 2256-2262.

(22) Kotaidis, V.; Dahmen, C.; von Plessen, G.; Springer, F.; Plech, A. J. Chem. Phys. 2006, 124, 184702.

(23) Pelton, M.; Liu, M.; Kim, H. Y.; Smith, G.; Guyot-Sionnest, P.; Scherer, N. F. Opt. Lett. 2006, 31, 2075-2077. 